\title{
Contribución de la bioenergía al desarrollo sostenible del medio rural. Análisis para Castilla y León
}

\author{
ANA QUIJANO PEDROSA \\ Fundación CARTIF.ESPAÑA. E-mail: anaqui@cartif.es \\ MARGARITA RICO GONZÁLEZ \\ Departamento de Ingeniería Agrícola y Forestal. UNIVERSIDAD DE VALLADOLID, \\ ESPAÑA.E-mail: mrico@iaf.uva.es
}

\begin{abstract}
RESUMEN
El progresivo deterioro socioeconómico de buena parte del territorio rural del interior español ha motivado la necesidad de aplicar medidas de acción que procuren la diversificación productiva de estos espacios. En este sentido, el sector de la bioenergía constituye una actividad susceptible de generar efectos económicos y sociales en las zonas de localización. El objetivo de este trabajo consiste en analizar los impactos que actualmente puede tener el sector de la bioenergía en las zonas rurales de Castilla y León, así como las potencialidades y los obstáculos para su correcto desarrollo. Los resultados obtenidos muestran que los importantes beneficios atribuidos a este sector no se están produciendo como era esperado y que aún hay que superar importantes trabas, tanto desde el punto de vista de la oferta como de la demanda, para que la bioenergía contribuya de manera generalizada al desarrollo del medio rural.
\end{abstract}

Palabras clave: Espacio rural, Diversificación económica, Bioenergía, Impactos socioeconómicos, Castilla y León.

\section{Bioenergy Contribution to Sustainable Rural Development. Analysis for Castilla y León}

\begin{abstract}
The progressive socioeconomic decline in Spanish rural areas has motivated the need for action measures to diversify production of these spaces. In this sense, the bioenergy sector is an activity likely to generate economic and social effects in the areas of location. The aim of this work is to analyze the possible impact of the bioenergy sector in rural areas in Castilla y León, as well as the potential and obstacles for its development. Results show that significant benefits attributed to this sector are not producing as expected and there are still significant problems, both from the point of view of supply and demand, impeding the contribution of bioenergy to rural development.
\end{abstract}

Keywords: Rural areas, Economic diversification, Bioenergy, Socioeconomic impacts, Castilla y León.

Clasificación JEL: O13, O18, R58

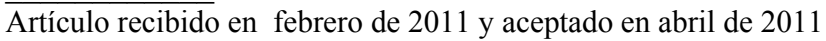

Artículo disponible en versión electrónica en la página www.revista-eea.net, ref. ə-29109 


\section{INTRODUCCIÓN ${ }^{1}$}

Las políticas de desarrollo rural puestas en marcha en los últimos años han planteado como uno de sus principales objetivos la diversificación de la economía de las zonas rurales, teniendo en cuenta el carácter multifuncional de estos espacios y, como consecuencia de ello, promocionando actividades económicas alternativas a la agricultura tradicional (Comisión Europea 1988, 1996, 2003; Moyano y Garrido, 2007). El fin último de estas estrategias de desarrollo consiste en dinamizar el tejido socioeconómico de gran parte del territorio rural del interior peninsular (Van Huylenbroeck y Durand, 2003; Rico y Gómez-Limón, 2008; González Regidor, 2008), intentando frenar así el incesante abandono poblacional y todo ello bajo el principio de sostenibilidad medioambiental ${ }^{2}$.

A este respecto, cabe destacar que el "Chequeo Médico" de la Política Agraria Común (PAC) del año $2009^{3}$ estableció como áreas prioritarias para ser financiadas por parte de los Estados Miembros, acciones relacionadas con el cambio climático, la gestión del agua, la biodiversidad o las energías renovables. Como consecuencia, la producción, aprovechamiento y transformación de biomasa con fines energéticos se configura en la actualidad como una actividad de especial relevancia en las zonas rurales (Rodríguez y Sánchez, 2008), ya que representa una nueva orientación a la agricultura tradicional, aprovechando los recursos endógenos del territorio y preservando el medio ambiente ${ }^{4}$, atendiendo así a las premisas europeas (Dincer, 2000).

De este modo, tanto los diversos planes de acción públicos de fomento de las energías renovables implementados en los últimos años, como la mayor parte de la literatura al respecto, han puesto de manifiesto el importante protagonismo que puede representar el sector de la bioenergía ${ }^{5}$ dentro de la actual dinámica

${ }^{1}$ Los autores agradecemos las sugerencias aportadas por los evaluadores anónimos, en la medida en que han permitido mejorar el contenido final del trabajo.

${ }^{2}$ Uno de los diez puntos de la Declaración de Cork (Comisión Europea, 1996) se basa en el desarrollo sostenible del medio rural, en el sentido de que ha de mantenerse la calidad y la función de los paisajes rurales de Europa, ya se trate de recursos naturales, biodiversidad o identidad cultural.

${ }^{3}$ Reglamento (CE) no 74/2009 del Consejo de 19 de enero de 2009.

${ }^{4}$ En este sentido, cabe apuntar que también existen opiniones contrarias a la utilización de la biomasa a gran escala para usos energéticos, principalmente provenientes de grupos ecologistas, argumentando que no son del todo ciertos los beneficios que tradicionalmente son atribuidos a este tipo de energía y proponiendo otro tipo de aprovechamientos de esa biomasa (Carpintero, 2006; Ecologistas Martxan, 2002).

${ }^{5}$ El término bioenergía se refiere al tipo de energía renovable que puede ser obtenida a partir de la biomasa. Por su parte, se entiende por biomasa cualquier material orgánico originado en un proceso biológico, espontáneo o provocado, de origen vegetal o animal, susceptible de ser utilizado como fuente de energía. 
del mundo rural (CES de Castilla y León, 2009), dados sus impactos potenciales tanto desde un punto de vista socioeconómico como ambiental (Burguillo y del Río, 2008; ADAS, 2003). La conexión del recurso con el medio rural posibilita la implantación de sistemas energéticos con un enfoque territorial, que podría garantizar la autosuficiencia energética, a la vez que económica, de las zonas rurales. Por otro lado, dada la necesidad del sector energético de encontrar combustibles alternativos renovables y ante una situación medioambiental preocupante, con problemas tan graves como el cambio climático, las energías renovables son contempladas como una posible solución, tanto en países desarrollados como en países en vías de desarrollo (Meier y Munasinghe, 2004; FAO, 2008).

A este respecto, la estrecha relación entre bioenergía y desarrollo rural es constatada a nivel internacional por parte de organismos tales como la Organización de las Naciones Unidas para la Agricultura y la Alimentación (FAO), que manifiesta la potencialidad de esta actividad para crear empleos y fuentes alternativas de ingresos para agricultores de países en desarrollo, cuya población está sometida en una gran proporción a problemas de inseguridad alimentaria (FAO, 2009). Por su parte, y a nivel nacional, el borrador del futuro Plan de Acción Nacional de Energías Renovables (PANER) para el periodo 2011-2020 ${ }^{6}$, menciona dentro de los múltiples beneficios de las energías renovables sobre la sociedad el desarrollo del medio rural. Finalmente, a nivel regional, el Plan Regional de Ámbito Sectorial de la Bioenergía de Castilla y León aprobado recientemente $(\mathrm{PBCyL})$, establece cinco principios rectores que dan existencia al Plan y que están estrechamente relacionados con la argumentación expuesta hasta el momento: a) Principio de desarrollo rural e interés social; b) Principio de visión de un desarrollo ambientalmente sostenible; c) Principio de diversificación energética como aportación al sector energético actual; d) Principio de desarrollo económico y empresarial y e) Principio de uso de los recursos de Castilla y León.

\section{OBJETIVOS Y METODOLOGÍA}

Una vez descritos los antecedentes en los que se basa la realización de este estudio, su objetivo se centra en analizar los impactos que actualmente puede tener el sector de la bioenergía sobre las zonas rurales, así como las potencialidades y los obstáculos para su correcto desarrollo.

Así, con el fin de integrar los aspectos medioambientales, bioenergéticos y de desarrollo rural, este estudio pretende conocer las expectativas que tienen un

\footnotetext{
${ }^{6}$ A fecha de elaboración del presente trabajo (enero de 2011) el PANER no ha sido objeto de aprobación, si bien las previsiones del Gobierno situaban su puesta en marcha para finales del año 2010 .
} 
nutrido grupo de expertos acerca del futuro del sector de la bioenergía en las zonas rurales, que permita concluir con una recopilación de las principales directrices a seguir para superar las barreras que actualmente frenen la implantación de proyectos de naturaleza bioenergética como fuente de ingresos en el medio rural. Como caso de estudio se ha seleccionado la Comunidad Autónoma de Castilla y León, una región que está padeciendo desde hace décadas los efectos del deterioro socioeconómico de sus zonas rurales y que además posee un claro potencial para el desarrollo del sector de la bioenergía (CES de Castilla y León, 2009; Rodríguez y Sánchez, 2008).

Para alcanzar el objetivo planteado, se ha empleado una metodología cualitativa basada en la realización de entrevistas semiestructuradas a un grupo de expertos relacionados con el sector de la bioenergía en Castilla y León. La selección de expertos ha sido meticulosa, con objeto de alcanzar la mayor representatividad posible y obtener así respuestas desde las distintas ópticas, intereses o expectativas que pudieran surgir dentro del sector analizado ${ }^{7}$. En concreto, se ha contado con un total de 12 expertos que pueden ser categorizados en cuatro grupos: a) Grupo de producción y logística de biomasa, donde han participado representantes de agricultores y representantes de asociaciones forestales; b) Grupo de producción y logística de bioenergía, compuesto por empresarios del sector e investigadores acerca de la producción de bioenergía; c) Grupo de planificación y gestión de la bioenergía, formado por técnicos que trabajan dentro de los servicios administrativos encargados de gestionar asuntos variados relacionados con la bioenergía y técnicos de agencias energéticas y d) Grupo de investigación universitaria sobre bioenergía y desarrollo rural, donde se encuentran profesores universitarios expertos en las líneas de investigación señaladas.

Como se ha apuntado, se ha realizado una entrevista personal semiestructurada a cada uno de estos expertos. Este tipo de entrevistas consisten en articular un proceso de interacción dinámica de comunicación entre el entrevistador y el entrevistado con el fin de obtener información lo más implicante posible sobre el objeto de análisis que se plantea. Adicionalmente, se trabaja con un guión que enumera el conjunto de preguntas predeterminadas o temas que se van a tratar, pero deja un gran margen de libertad a la persona entrevistada para que refleje su punto de vista de la manera más abierta posible. Por lo tanto, el cuestionario empleado contiene un conjunto de preguntas de tipo cerrado (con posibilidades de contestación prefijadas) pero deja un margen de libertad para que el experto consultado añada todo aquello que considere

\footnotetext{
${ }^{7} \mathrm{Si}$ bien se ha intentado seleccionar un panel de expertos lo más representativo posible del sector analizado, la subjetividad de sus respuestas inherente a las técnicas metodológicas basadas en la recopilación de opiniones y percepciones individuales, puede condicionar los resultados finalmente obtenidos, tal y como ocurre en todos los estudios de este tipo.
} 
oportuno. A este respecto, el contenido temático del cuestionario se ha estructurado del siguiente modo: el primer bloque cuestiona a los expertos acerca de la repercusión de la bioenergía sobre el desarrollo de las zonas rurales de Castilla y León. En el segundo bloque se ha consultado acerca de los impactos concretos que este sector podría generar sobre el medio rural de la región en el caso de su implantación generalizada. El tercer y último bloque de preguntas recaba información acerca de las potencialidades y los obstáculos para dinamizar las zonas rurales a través del sector de la bioenergía, tanto desde el punto de vista de la oferta del recurso como de su demanda y también se consulta sobre las estrategias más adecuadas para superar las trabas existentes en la actualidad.

Una vez expuesta la introducción, los objetivos y la metodología que va a seguir este trabajo, en el capítulo tercero se detallarán algunos datos que caracterizan actualmente al sector de la bioenergía en Castilla y León y su interrelación con las zonas rurales. En el capítulo cuarto se ofrecerán los principales resultados extraídos a partir de las entrevistas hechas al conjunto de expertos seleccionados. El capítulo quinto está dedicado a proponer una serie de estrategias para favorecer el desarrollo efectivo de proyectos bioenergéticos en el medio rural. Finalmente, el capítulo sexto sintetiza las conclusiones del estudio.

\section{EL SECTOR DE LA BIOENERGÍA EN CASTILLA Y LEÓN}

Aunque las propiedades de la biomasa para la producción de bioenergía son conocidas desde hace tiempo, el desarrollo de los combustibles fósiles ha desplazado su papel como fuente energética prioritaria. Con el tiempo y ante el temido agotamiento de los combustibles fósiles, la biomasa ha vuelto a recuperar importancia en el sector energético. Iniciativas como el Protocolo de Kioto y el desarrollo de otras normativas en el ámbito de la Unión Europea y nacional, también han favorecido la sustitución progresiva de los combustibles habituales, con el fin de garantizar el abastecimiento energético futuro y preservar el medio ambiente. En este apartado se analiza de manera comparativa la utilización de bioenergía en Castilla y León, así como su potencial futuro y se describe la estrecha interrelación entre el desarrollo de este sector y el desarrollo del mundo rural.

\subsection{Contribución de la biomasa en el mix energético}

El sector energético de Castilla y León se basa en el uso de combustibles no renovables, lo cual requiere tener que importar gran parte de la energía consumida, ya que más del $90 \%$ del consumo de energía primaria procede de energía fósil, mientras que sólo el $7 \%$ es de origen renovable (Tabla 1). Esta desproporción entre el consumo de energía fósil y renovable se repite de igual 
modo a nivel mundial, europeo y nacional, aportando las renovables respectivamente el $13 \%, 8 \%$ y $7 \%$ del total de la energía primaria consumida en dichos ámbitos territoriales. Por su parte, y dentro del consumo de energía primaria renovable en Castilla y León, la energía hidráulica es la más representativa, seguida de la bioenergía y la eólica. Esta situación difiere de lo acontecido a nivel nacional, donde la biomasa es la principal fuente energética renovable alcanzando cifras de consumo mayores que la energía hidráulica. Por último, cabe destacar que en esta región el uso mayoritario que se le da a la biomasa es en forma de energía térmica (62\%), seguido de la eléctrica $(26 \%)$ y el transporte (12\%), unos usos más equilibrados que lo que acontece a nivel nacional, donde los usos térmicos alcanzan el $70 \%$, mientras que los eléctricos y los de transporte tan solo el $23 \%$ y el $7 \%$ respectivamente.

Tabla 1

Análisis comparativo del contexto energético en Castilla y León, 2010

\begin{tabular}{|c|c|c|c|c|}
\hline & MUNDO & UE27 & ESPAÑA & $\begin{array}{c}\text { CASTILLA Y } \\
\text { LEÓN }\end{array}$ \\
\hline $\begin{array}{c}\text { Producción de energía } \\
\text { primaria (Mtep/año) }\end{array}$ & 12.000 & 850 & 31 & 5 \\
\hline $\begin{array}{c}\text { Consumo de energía } \\
\text { primaria (Mtep/año) }\end{array}$ & 12.000 & 1.800 & 147 & 10 \\
\hline $\begin{array}{c}\text { Distribución del } \\
\text { consumo de energía } \\
\text { primaria en fósil - } \\
\text { nuclear - renovable } \\
\text { (\%) }\end{array}$ & $81-6-13$ & $79-13-8$ & $83-10-7$ & $92-1-7$ \\
\hline $\begin{array}{c}\text { Distribución del } \\
\text { consumo de energía } \\
\text { primaria renovable en } \\
\text { hidráulica - eólica - } \\
\text { solar - geotermia - } \\
\text { bioenergía (\%) }\end{array}$ & $18-3-1-1-77$ & $19-7-1-4-69$ & $23-23-1-<1-53$ & $49-20-3-<1-28$ \\
\hline $\begin{array}{c}\text { Distribución del } \\
\text { consumo de energía } \\
\text { primaria de biomasa } \\
\text { en térmica - eléctrica - } \\
\text { transporte (\%) }\end{array}$ & - & $50-37-13$ & $70-23-7$ & $62-26-12$ \\
\hline
\end{tabular}

Fuente: Junta de Castilla y León (2010).

A este respecto, el Plan de las Energías Renovables Español (PER), vigente para el periodo 2005-2010, establece una serie de medidas y objetivos a alcanzar a nivel nacional con el propósito de fomentar el uso de energías renovables y contribuir así con los compromisos internacionales (Protocolo de Kioto) y europeos. Así, el PER propuso lograr un abastecimiento energético a partir de energías renovables del $12,1 \%$, una producción eléctrica con fuentes 
no fósiles de un 30,3\% del consumo eléctrico bruto y un consumo de biocarburantes del 5,83\%, recayendo gran parte de estos objetivos en la biomasa. El grado de logro alcanzado podrá conocerse próximamente, tras llevarse a cabo un análisis exhaustivo del periodo una vez finalizada la vigencia del Plan. Sin embargo, hay que mencionar que las expectativas de cumplimiento de dichos objetivos son bastante pesimistas, ya que según un informe elaborado por la Comisión Europea (Comisión Europea, 2009) se desprende que en el año 2007 ni Europa ni España estaban realizando los avances necesarios para alcanzar las cuotas de uso de bioenergía consignadas, con lo que muy presumiblemente no se hayan logrado los objetivos propuestos para el año 2010.

Para el horizonte 2020 la Unión Europea ha previsto una nueva normativa ${ }^{8}$ en la que se recogen tanto objetivos globales nacionales obligatorios como medidas para el uso de la energía procedente de fuentes renovables. Dichos retos se resumen en: conseguir en el año 2020 un consumo de energías renovables del $20 \%$, ahorrar un $20 \%$ del consumo energético y reducir las emisiones de efecto invernadero en un $20 \%$ con respecto a 1990. En cuanto a los objetivos a alcanzar en el sector del transporte, la meta deseada es un consumo de biocarburantes del 10\%. Por su parte, el PANER correspondiente al periodo 2011-2020 se ha elaborado con el fin de lograr los objetivos nacionales fijados en la normativa europea y contiene estimaciones de consumo de bioenergía para el año 2020 que incluso superan los objetivos previstos por la Unión Europea anteriormente especificados.

Para el caso de Castilla y León, el PBCyL analiza los recursos de biomasa de la región susceptibles de valorización energética y sus posibles aplicaciones y establece medidas específicas y objetivos a alcanzar dentro del periodo 20102020, configurándose como el primer plan a nivel regional a favor de la bioenergía en España.

\subsection{Potencial y perspectiva futura del consumo de biomasa}

La planificación de los ambiciosos objetivos a alcanzar tanto en el PANER como en el PBCyL, es fruto del gran potencial que poseen las energías renovables y en concreto de las posibilidades que ofrece el recurso de la biomasa (ASEMFO, 2007; CES de Castilla y León, 2009; APPA, 2010). Así, según los cálculos realizados para la elaboración del $\mathrm{PBCyL}$, la producción potencial de biomasa en Castilla y León se estima en 78.894.600 t/año (16.344 ktep/año) para el año 2020, mientras que la producción de biomasa fácilmente

\footnotetext{
${ }^{8}$ Directiva 2009/28/CE del Parlamento Europeo y del Consejo de 23 de abril relativa al fomento del uso de energía procedente de fuentes renovables por la que se modifican y derogan las Directivas 2001/77/CE y 2003/30/CE.
} 
valorizable es de 8.440 .580 t/año (1.788 ktep/año). Teniendo en cuenta de manera individual cada tipo de biomasa (forestal, agrícola, ganadera, industria de la madera, etc.), el mayor reto en la actualidad para esta Comunidad Autónoma será valorizar tanto los cultivos energéticos como los residuos forestales y agrícolas.

Si bien el uso de biocombustibles en España y en Castilla y León está lejos de la situación de otros países como Suecia, Alemania o Italia, el sector de la bioenergía ha experimentado en los últimos años un claro crecimiento. Esta situación se ha visto impulsada gracias al desarrollo de planes de acción y normativa para el fomento del mercado de biomasa, así como a la investigación llevada a cabo con objeto de optimizar la logística de este recurso y resolver algunas barreras técnicas de uso de algunos tipos de biomasa.

No obstante, existen diferencias en cuanto al desarrollo registrado por los diferentes usos de la biomasa. Así, mientras la biomasa térmica ha mostrado un crecimiento importante, la biomasa eléctrica y el uso de biocarburantes han experimentado una expansión menor. El crecimiento de los usos de la biomasa para fines térmicos se ha basado principalmente en el aumento del número de instalaciones en hogares y edificios públicos, aunque también las industrias de los sectores agrario y maderero han incorporado un número importante de calderas de biomasa dada la abundancia de materia prima existente en su entorno.

Por su parte, la producción eléctrica con biomasa comenzó a desarrollarse tras la mejora de las condiciones de remuneración a las energías renovables en el año 2007. Desde entonces en Castilla y León se ha planificado la construcción de varias plantas eléctricas e incluso alguna ya está en funcionamiento. Del mismo modo, la utilización de biogas como fuente energética ha comenzado a explotarse de manera reciente en vertederos, depuradoras, pequeñas explotaciones agrícolas y en centros de tratamiento de residuos. Finalmente, las plantas de biocarburantes comienzan a extenderse a lo largo de la Comunidad, existiendo en la actualidad tres plantas de producción de biodiésel y una planta de bioetanol.

\subsection{Bioenergía y desarrollo rural}

Bajo la amenaza del continuo despoblamiento de las zonas rurales (García Pascual, 2003; Gómez-Limón et al., 2007), las prioridades de un proceso de recuperación del medio rural han de centrarse en los aspectos socioeconómicos $\mathrm{y}$, particularmente, en la creación de empleo que favorezca la fijación de población (García Sanz e Izcara, 2000; Brouwer, 2004). Esta es una de las principales razones por las cuales se considera que el sector de la bioenergía puede contribuir a mejorar el medio rural, teniendo en cuenta los efectos económicos, sociales y ambientales que generan este tipo de actividades sobre 
el territorio (Burguillo y del Río, 2008; del Río y Burguillo, 2008). Al hacer referencia a la biomasa, hay que subrayar la importante conexión del recurso con el medio rural, ya que es su principal fuente productora y de ahí que los proyectos de revalorización energética tengan siempre implícita una mejora del sector agrario del territorio en el que se ubican (Franco, 2007; Rodríguez y Sánchez, 2008). Por otra parte, también hay que mencionar una serie de beneficios indirectos o efectos de arrastre asociados a estas actividades que consiguen que el impacto en la creación de empleo sea mayor.

Según Burguillo y del Río (2008) los impactos que la implantación de la bioenergía puede generar sobre el desarrollo sostenible del medio rural se determinan teniendo en cuenta las tres dimensiones de la sostenibilidad: dimensión económica, social y ambiental (Figura 1). El análisis de esos tres conjuntos de efectos de la bioenergía sobre el territorio representa un estudio clave para justificar la efectiva contribución de dicho sector al desarrollo sostenible de las áreas rurales (ADAS, 2003).

Figura 1

Impacto de la bioenergía sobre el desarrollo rural sostenible

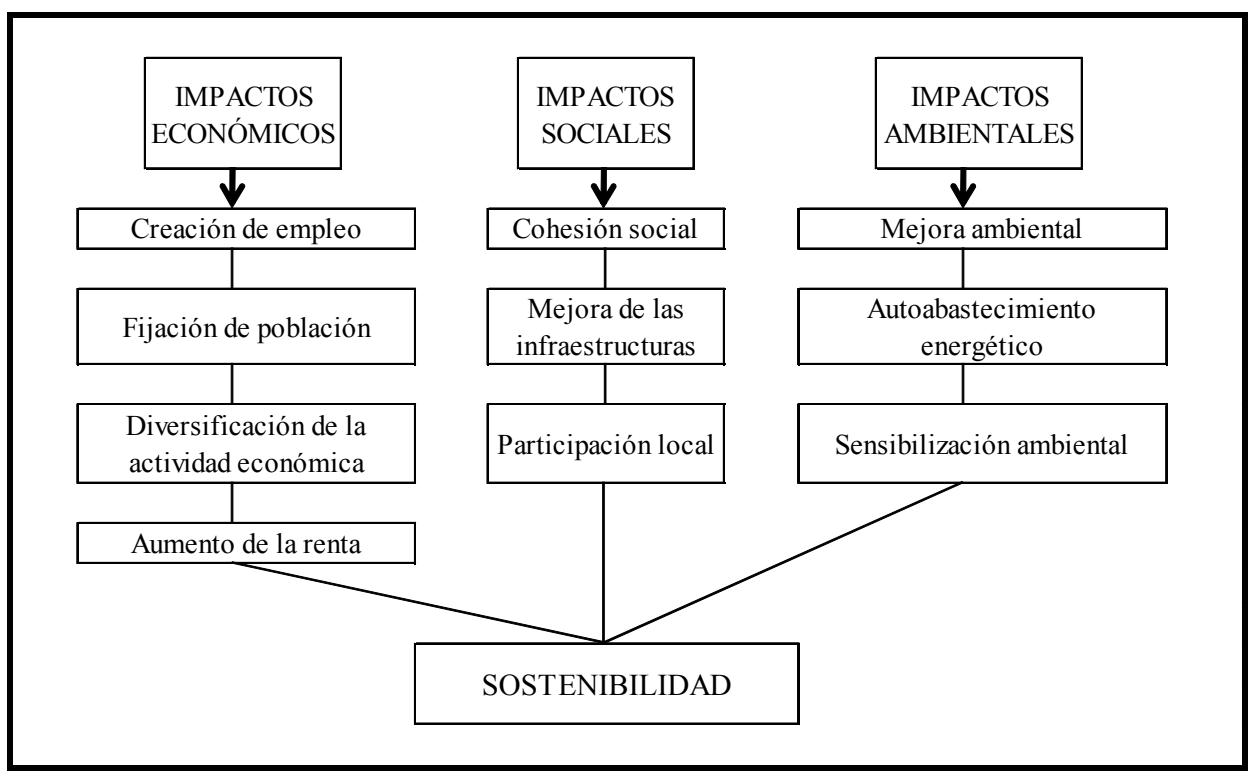

Fuente: Elaboración propia a partir de Burguillo y del Río (2008).

Desde la óptica de los impactos económicos, los efectos sobre la creación de empleo tanto cuantitativa como cualitativamente se consideran como los más representativos. Así, por ejemplo, en el PER se estimó la creación de 
aproximadamente 100.000 empleos netos en el sector de las renovables durante el periodo 2005-2010, de los cuales 56.000 debieran corresponderse al aprovechamiento energético de la biomasa, refiriéndose tanto a los relativos a la inversión en la implantación de los proyectos como de la explotación de los mismos. En un estudio de la Asociación de Productores de Energías Renovables (APPA, 2010) se revela que en el año 2009 el total de empleos directos en España en ese sector ascendía a 59.303 personas, mientras que el empleo indirecto suponía un total de 40.547 ocupados (un total de 99.850 puestos de trabajo). Del total de empleos directos, el $43 \%$ corresponde a los subsectores de los biocombustibles y la biomasa, lo que da cuenta de la capacidad de generación de empleo de la bioenergía. Por su parte, algunos autores han mostrado a través de estudios de caso en diferentes comunidades autónomas españolas la efectiva creación de empleo a través de iniciativas empresariales en este sector (Faulin et al., 2006, Míguez et al., 2006 o López et al., 2006). Este aumento del empleo implicaría una mejora sobre la situación demográfica de las zonas rurales donde se asentaran plantas de biomasa en forma de aumento de la población y rejuvenecimiento de la misma. Este impacto resulta de vital importancia en áreas con especiales problemas poblacionales, donde el éxodo demográfico y el envejecimiento están comprometiendo la estructura socioeconómica de gran parte del territorio rural del interior español y de las zonas de montaña (Fundación Encuentro, 2007).

Asimismo, y dentro de los efectos económicos, es necesario hablar de los impactos que supondrían las inversiones de bioenergía sobre la diversificación del tejido productivo de las zonas rurales. En concreto, sobre el sector primario podría fomentarse un cambio de orientación productiva hacia cultivos con fines energéticos cuya venta en el mercado estaría asegurada en las plantas transformadoras. El gran potencial de biomasa procedente del sector forestal también permitiría valorizar este recurso, muy subaprovechado en muchas zonas forestales españolas (Ortuño et al., 2010; ASEMFO, 2007). Por otro lado, el fomento del sector de la bioenergía daría lugar a la creación de instalaciones industriales (producción de biodiesel, bioetanol, pelets, etc.) y el consiguiente avance del sector secundario en las zonas rurales, junto con todas las empresas de apoyo que podrían crearse. Siempre y cuando estas vinculaciones recaigan sobre agentes de la zona, las repercusiones sobre la creación de empleo rural, el aumento de la renta de la población local y la diversificación productiva en la zona serán mayores. Los efectos sobre el sector terciario consistirían en la dinamización de empresas de transporte o comerciales, junto con los impactos positivos que podría tener sobre el turismo rural y sobre la administración pública (construcción de infraestructuras gracias a los ingresos en la arcas municipales derivados de los proyectos bioenergéticos...).

En lo referente a impactos de tipo social, varios estudios señalan el mayor grado de cohesión que la población rural puede alcanzar gracias a proyectos de 
este tipo, derivados de su participación en el proceso de implantación de la iniciativa, del incremento del movimiento asociativo y cooperativo y de los acuerdos que podrían realizarse entre los distintos actores socioeconómicos locales (Franco, 2007; del Río y Burguillo, 2008).

Finalmente, el impacto medioambiental positivo es una de las principales justificaciones para la promoción de este tipo de proyectos (Rodríguez y Sánchez, 2008). Al analizar este impacto a escala local, aparte de los beneficios que puedan producirse sobre el medio ambiente del territorio, hay que hablar de otros efectos relacionados con la mayor concienciación ecológica de la población de la zona, con la mejor imagen del territorio o con la mayor educación ambiental de los habitantes. Sin embargo, es necesario también apuntar la posible existencia de efectos medioambientales indeseables, relacionados con los residuos, emisiones, uso del suelo o impactos paisajísticos negativos derivados del asentamiento de las plantas transformadoras.

\section{BIOENERGÍA Y DESARROLLO RURAL EN CASTILLA Y LEÓN: LA OPINIÓN DE LOS EXPERTOS}

Tal y como se especificó en el apartado metodológico, la parte empírica de este trabajo ha consistido en consultar a un grupo de expertos relacionados con la bioenergía acerca de la repercusión de este sector sobre el desarrollo rural, los impactos potenciales que podrían generarse sobre el territorio y las posibilidades y limitaciones actuales para su correcto desarrollo. A continuación se detallan los resultados obtenidos.

\subsection{Repercusión de la bioenergía sobre el desarrollo rural}

En este apartado se ha cuestionado a los expertos sobre la posible necesidad de conservar el medio rural y sobre cuáles son los sectores económicos que pueden sustentar el agro en el futuro y se ha evaluado la posibilidad de contribuir en la mejora del medio rural mediante la bioenergía. Por último, se ha determinado la sinergia entre las políticas de desarrollo rural y las políticas energéticas.

Al hilo de la estructura expuesta, en primer lugar cabe señalar que la mayor parte de expertos entrevistados consideran que es "muy necesario" conservar el medio rural y aplicar medidas públicas para alcanzar tal fin. El 16,7\% considera que es "bastante necesario", mientras que ninguno de los expertos con los que se ha conversado han manifestado que es "algo", "poco" o "nada necesario" conservar las zonas rurales.

A continuación, el cuestionario planteaba la valoración de varias alternativas económicas como base del futuro del medio rural, teniendo en cuenta que las políticas de desarrollo rural surgidas en los últimos años tienen como objetivo 
prioritario la diversificación de actividades productivas en estas áreas. Así, utilizando una escala Likert de 1 a 5 (1=nada de acuerdo con que esa actividad pueda ser la base económica futura del medio rural y $5=$ muy de acuerdo), los expertos consultados consideraron a las actividades del sector primario innovador (producciones de calidad, biomasa, productos ecológicos, etc.) como la alternativa económica principal de cara al futuro de las zonas rurales de Castilla y León, con una valoración media de 4,18 puntos sobre la escala referenciada. Las actividades más valoradas a continuación han sido: la industria agroalimentaria $(3,35)$, el turismo rural $(3,03)$ y el sector primario tradicional $(2,71)$. Han quedado en un plano más residual los servicios a la población $(2,64)$, el territorio rural como espacio de residencia $(2,47)$ y el sector industrial, a excepción de la industria agroalimentaria $(1,75)$.

Por otro lado, el análisis a las respuestas relacionadas con la opinión de los expertos sobre el beneficio socioeconómico que podría representar el sector de la bioenergía sobre los espacios rurales, confirma las expectativas puestas en este tipo de actividades, ya que un $66,7 \%$ de los entrevistados afirmaron estar "bastante" y "muy de acuerdo" en considerar a la bioenergía como una alternativa económica favorecedora del medio rural (Tabla 2).

Tabla 2

La bioenergía como alternativa para promocionar el desarrollo rural

\begin{tabular}{|c|c|c|c|c|}
\hline $\begin{array}{c}\text { Nada de } \\
\text { acuerdo }\end{array}$ & $\begin{array}{c}\text { Poco de } \\
\text { acuerdo }\end{array}$ & $\begin{array}{c}\text { Algo de } \\
\text { acuerdo }\end{array}$ & $\begin{array}{c}\text { Bastante de } \\
\text { acuerdo }\end{array}$ & $\begin{array}{c}\text { Muy de } \\
\text { acuerdo }\end{array}$ \\
\hline $0 \%$ & $0 \%$ & $33,3 \%$ & $41,7 \%$ & $25 \%$ \\
\hline
\end{tabular}

Fuente: Elaboración propia a partir de los resultados de los cuestionarios.

Finalmente, dentro de este apartado se indagó acerca de la opinión de los expertos sobre el conjunto de políticas de desarrollo rural y su apoyo a la bioenergía, así como sus opiniones sobre la consideración del desarrollo rural dentro de las políticas bioenergéticas. A este respecto, y a tenor de los datos de la Tabla 3, parece desprenderse que los expertos mantienen un cierto descontento en torno al apoyo al sector de la bioenergía dentro de las políticas públicas de desarrollo rural. Alguna de las personas que participaron en el estudio confirmaron la importancia de implantar nuevas medidas que permitan impulsar el sector de la bioenergía dentro de las zonas rurales de esta región y que este sector pase de ser una promesa a convertirse en una realidad. Por su parte, los expertos consideran en una mayor proporción que las actuales políticas energéticas, dentro de su apartado dedicado a la bioenergía, sí que están contribuyendo en parte a la dinamización de los espacios rurales de la región. 
Tabla 3

Opinión sobre las políticas de desarrollo rural y las políticas bioenergéticas

\begin{tabular}{|l|c|c|c|c|c|}
\hline & $\begin{array}{c}\text { Nada de } \\
\text { acuerdo }\end{array}$ & $\begin{array}{c}\text { Poco de } \\
\text { acuerdo }\end{array}$ & $\begin{array}{c}\text { Algo de } \\
\text { acuerdo }\end{array}$ & $\begin{array}{c}\text { Bastante } \\
\text { de } \\
\text { acuerdo }\end{array}$ & $\begin{array}{c}\text { Muy de } \\
\text { acuerdo }\end{array}$ \\
\hline $\begin{array}{l}\text { Las actuales políticas de } \\
\text { desarrollo rural contribuyen } \\
\text { al fomento de la bioenergía }\end{array}$ & $8,3 \%$ & $58,3 \%$ & $25,0 \%$ & $8,3 \%$ & $0,0 \%$ \\
\hline $\begin{array}{l}\text { Las actuales políticas de } \\
\text { bioenergía contribuyen al } \\
\text { desarrollo del medio rural }\end{array}$ & $0,0 \%$ & $25,0 \%$ & $50,0 \%$ & $25,0 \%$ & $0,0 \%$ \\
\hline
\end{tabular}

Fuente: Elaboración propia a partir de los resultados de los cuestionarios.

\subsection{Impactos de la bioenergía en el desarrollo rural}

En este apartado se han recogido las opiniones de los expertos entrevistados acerca de los impactos que puede generar el sector de la bioenergía sobre la dinamización del medio rural desde diversas ópticas. Para ello, y tal y como puede observarse en la Tabla 4, se ha mostrado a cada uno de los expertos una batería de posibles efectos que podría generar el establecimiento de plantas de bioenergía y su consumo sobre las zonas rurales, desde un punto de vista socioeconómico (efectos sobre el empleo, la demografía, la actividad económica, las infraestructuras y la vida social) y también desde una visión meramente ambiental. Cada uno de estos efectos debían ser valorados en una escala Likert que variaba entre 1 (impacto nulo) y 5 (gran impacto) dependiendo de los impactos potenciales que se estimaran.

Consecuentemente, la primera conclusión general que puede desprenderse tras el análisis efectuado en este apartado, es la apreciación general de la existencia de una influencia positiva en el medio rural derivada de la implantación de instalaciones de aprovechamiento energético de biomasa, ya que el impacto medio alcanza un total de 3,52 puntos de un máximo de 5 . Así, y según los grandes bloques de impactos estudiados, la mayor repercusión positiva que la bioenergía puede ocasionar en el medio rural recae sobre el medio ambiente, mientras que los menores impactos se detectan en los aspectos demográficos y sociales.

Dentro de las cuestiones socioeconómicas, la creación de empleo es el impacto más valorado, asociándose la implantación de instalaciones de bioenergía en el medio rural con un empleo de pobladores rurales y duradero. Asimismo, es sustancial la valoración que los expertos hacen acerca de los efectos positivos sobre la diversificación económica (condición necesaria para la revitalización rural, tal y como postulan las políticas de desarrollo rural) y sobre la posible mejora del sector primario, dado que la mayor parte de materia prima utilizada se estima que sería de la zona de ubicación de la industria. No 
obstante, parece percibirse que el desarrollo del sector de la bioenergía no repercutiría tan positivamente sobre otras variables socioeconómicas que actualmente registran unas cifras muy preocupantes en el medio rural castellano y leonés, como es la tasa de actividad femenina, la evolución cuantitativa de la población o la tasa de juventud en estos espacios.

Tabla 4

Impactos de la bioenergía sobre el desarrollo rural

\begin{tabular}{|c|c|c|c|c|}
\hline & $\begin{array}{l}\text { Tipo de impacto } \\
\text { (media) }\end{array}$ & Descripción del impacto & Media & $\begin{array}{l}\text { Desv. } \\
\text { Típica }\end{array}$ \\
\hline \multirow{17}{*}{ 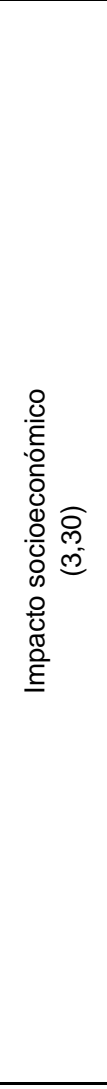 } & \multirow{7}{*}{$\begin{array}{c}\text { EMPLEO } \\
(3,49)\end{array}$} & Creación de empleo & 3,83 & 0,93 \\
\hline & & Empleo duradero & 3,91 & 0,66 \\
\hline & & Empleo para mujeres & 2,83 & 1,02 \\
\hline & & Empleo para jóvenes & 3,66 & 0,88 \\
\hline & & Empleo cualificado & 3,08 & 0,79 \\
\hline & & Empleo para población rural & 4,08 & 0,90 \\
\hline & & Traslado de empleo de otras actividades & 3,08 & 0,66 \\
\hline & \multirow{2}{*}{$\begin{array}{l}\text { FIJACIÓN DE } \\
\text { POBLACIÓN } \\
(3,07)\end{array}$} & Aumento de población rural & 2,91 & 1,31 \\
\hline & & Rejuvenecimiento de la población rural & 3,25 & 1,42 \\
\hline & \multirow{4}{*}{$\begin{array}{l}\text { ACTIVIDAD } \\
\text { ECONÓMICA } \\
(3,58)\end{array}$} & $\begin{array}{c}\text { Aumento considerable de explotaciones } \\
\text { dedicadas a cultivos energéticos de la } \\
\text { zona }\end{array}$ & 3,91 & 1,08 \\
\hline & & $\begin{array}{l}\text { Utilización mayoritaria de materia prima } \\
\text { procedente de la zona }\end{array}$ & 4,08 & 1,12 \\
\hline & & Aumento del turismo & 2,16 & 0,71 \\
\hline & & $\begin{array}{l}\text { Diversificación económica debido a las } \\
\text { necesidades de este tipo de industria }\end{array}$ & 4,16 & 0,57 \\
\hline & $\begin{array}{c}\text { INFRAESTRUCTURAS } \\
(3,25)\end{array}$ & $\begin{array}{l}\text { Mejora de las infraestructuras de } \\
\text { comunicaciones y servicios }\end{array}$ & 3,25 & 1,13 \\
\hline & \multirow{3}{*}{$\begin{array}{c}\text { SOCIAL } \\
(3,13)\end{array}$} & $\begin{array}{c}\text { Mayor cohesión social (acuerdos entre } \\
\text { la empresa energética y otras } \\
\text { instituciones) }\end{array}$ & 3,50 & 0,79 \\
\hline & & Mayor distribución de la renta & 3,41 & 0,99 \\
\hline & & $\begin{array}{l}\text { Acogida positiva de la población rural } \\
\text { hacia este tipo de proyectos }\end{array}$ & 3,50 & 1,00 \\
\hline \multirow{3}{*}{ 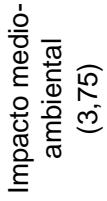 } & \multicolumn{2}{|c|}{ Mejora ambiental en la zona } & 3,91 & 0,89 \\
\hline & \multicolumn{2}{|c|}{ Autoabastecimiento de energía renovable } & 3,83 & 0,71 \\
\hline & \multicolumn{2}{|c|}{ Mayor sensibilización ambiental de la población } & 3,50 & 0,84 \\
\hline
\end{tabular}

Fuente: Elaboración propia a partir de los resultados de los cuestionarios. 


\subsection{Potencialidades y obstáculos al desarrollo del sector de la bioenergía}

Este apartado está orientado a determinar, según los expertos consultados, cuáles son las ventajas así como las barreras existentes para que el sector de la bioenergía se desarrolle convenientemente, lo que favorecerá o dificultará la revitalización del medio rural a través de esta actividad económica. Para ello, se han planteado varias cuestiones tanto desde el punto de vista de la producción de bioenergía como de su consumo, utilizando para su valoración también una escala Likert que oscila entre 1 (menor relevancia) y 5 (mayor relevancia).

\subsubsection{Desde el punto de vista de la oferta}

Al analizar este apartado, es preciso señalar que se han estudiado tanto los factores que influyen sobre la oferta de producción primaria (cultivos energéticos, biomasa forestal...) como sobre la oferta de producción secundaria (producto transformado). Así, atendiendo en primer lugar a los obstáculos detectados que frenan el desarrollo de este sector en el medio rural, el más influyente para los expertos consultados ha sido la desconfianza de los productores tanto primarios como secundarios por la falta de un verdadero mercado de bioenergía, como puede comprobarse en la Tabla 5. A continuación, el obstáculo que se ha considerado más relevante ha sido el desconocimiento de las oportunidades del sector de la bioenergía por parte tanto de agricultores como de posibles empresas promotoras. Por su parte, los obstáculos señalados como de menor importancia han sido la necesidad de invertir y adquirir nuevos hábitos por parte de los agricultores y la ausencia de primas a los cultivos energéticos en la PAC. Ya que la pregunta tenía también la posibilidad de contestar en formato abierto, es necesario apuntar otros obstáculos valorados como muy determinantes por algunos de los expertos y que no habían sido tenidos en cuenta en el cuestionario en primera instancia. Estos obstáculos son: la inexistencia de un marco normativo estable que regule el sector, el insuficiente apoyo institucional hacia la promoción de la bioenergía (falta de compromiso público para alcanzar los acuerdos internacionales adquiridos sobre consumo de biomasa), la falta de aplicación en ocasiones de los resultados de la investigación (mayor promoción de biocombustibles de segunda y tercera generación) y la escasa rentabilidad del aprovechamiento de la biomasa forestal asociada a los altos costes de extracción que precisan de una ayuda económica externa. 
Tabla 5

Obstáculos que condicionan la oferta de bioenergía

\begin{tabular}{|l|c|c|}
\hline \multicolumn{1}{|c|}{ Obstáculos } & $\begin{array}{c}\text { Valoración } \\
\text { media }\end{array}$ & $\begin{array}{c}\text { Desviación } \\
\text { típica }\end{array}$ \\
\hline $\begin{array}{l}\text { Ausencia actual de prima por parte de la PAC a los cultivos } \\
\text { energéticos }\end{array}$ & 1,19 & 0,60 \\
\hline $\begin{array}{l}\text { Desconocimiento de las oportunidades del sector de la biomasa } \\
\text { por parte de agricultores y empresas promotoras }\end{array}$ & 2,82 & 0,98 \\
\hline $\begin{array}{l}\text { Necesidad de invertir y adquirir nuevos hábitos en la preparación } \\
\text { de los cultivos }\end{array}$ & 2,00 & 1,18 \\
\hline $\begin{array}{l}\text { Desconfianza en el sector por falta de un verdadero mercado de } \\
\text { bioenergía }\end{array}$ & 3,37 & 0,81 \\
\hline
\end{tabular}

Fuente: Elaboración propia a partir de los resultados de los cuestionarios.

En el caso de los incentivos que pueden contribuir al desarrollo de la biomasa desde el punto de vista de la oferta (Tabla 6), el análisis efectuado determinó los siguientes resultados a partir de las valoraciones proporcionadas por los expertos consultados. La medida valorada con mayor puntuación media ha sido el establecimiento de una instalación en la propia zona que favorezca la creación de una logística de suministro, junto con la realización de contratos de provisión de materias primas con los agricultores, si bien existe una marcada disparidad en las respuestas de los expertos entrevistados. De este hecho cabe desprenderse que en general los expertos consideran el modelo de pequeñas plantas de energía a escala local como el más apropiado para favorecer tanto la oferta como la demanda de este tipo de energía y, por ende, para generar renta y empleo en las zonas rurales donde se ubique.

Conviene también señalar que nuevamente, y al contrario de lo que se podía esperar, los expertos otorgan escasa importancia a la incorporación de ayudas a cultivos energéticos por parte de la PAC. Este hecho puede deberse a la amplia heterogeneidad del grupo de expertos analizado, entre los cuales tan solo se encontraba un representante del sector agrícola.

Tabla 6

Incentivos que podrían favorecer la oferta de bioenergía

\begin{tabular}{|l|c|c|}
\hline \multicolumn{1}{|c|}{ Incentivos } & $\begin{array}{c}\text { Valoración } \\
\text { media }\end{array}$ & $\begin{array}{c}\text { Desviación } \\
\text { típica }\end{array}$ \\
\hline Incorporación de ayudas a cultivos con fines energéticos en la PAC & 1,00 & 1,09 \\
\hline $\begin{array}{l}\text { Formación y difusión en tipos de cultivos y necesidades de riego y } \\
\text { fertilización y sobre los beneficios socioeconómicos de la biomasa }\end{array}$ & 1,19 & 1,47 \\
\hline $\begin{array}{l}\text { Establecimiento de una instalación en la propia zona que favorezca } \\
\text { la creación de una logística de suministro }\end{array}$ & 2,46 & 1,69 \\
\hline $\begin{array}{l}\text { Realización de contratos de suministro de materia prima con los } \\
\text { agricultores por periodos largos y precios fijos }\end{array}$ & 2,46 & 1,36 \\
\hline
\end{tabular}

Fuente: Elaboración propia a partir de los resultados de los cuestionarios. 


\subsubsection{Desde el punto de vista de la demanda}

A tenor de las respuestas proporcionadas por los expertos en este apartado, los motivos principales por los que los consumidores no se deciden a emplear la bioenergía como fuente energética habitual están relacionados con el desconocimiento de las posibilidades que ofrece el recurso, así como por el mayor precio de las instalaciones bioenergéticas junto con el insuficiente apoyo público en forma de ayuda financiera que promocione su utilización. Los expertos añadieron algún otro obstáculo dentro de la pregunta abierta, pero estaba estrechamente relacionado con los ítems que aparecen en la Tabla 7.

Tabla 7

Obstáculos que condicionan la demanda de bioenergía

\begin{tabular}{|l|c|c|}
\hline \multicolumn{1}{|c|}{ Obstáculos } & $\begin{array}{c}\text { Valoración } \\
\text { media }\end{array}$ & $\begin{array}{c}\text { Desviación } \\
\text { típica }\end{array}$ \\
\hline La comodidad del uso de energía de origen fósil (gasoil...) & 2,58 & 1,08 \\
\hline $\begin{array}{l}\text { El desconocimiento de las posibilidades de la biomasa para } \\
\text { usos domésticos }\end{array}$ & 3,25 & 0,86 \\
\hline $\begin{array}{l}\text { El mayor precio de las instalaciones de biomasa junto con la } \\
\text { práctica inexistencia de ayudas que fomenten el consumo }\end{array}$ & 3,17 & 0,83 \\
\hline
\end{tabular}

Fuente: Elaboración propia a partir de los resultados de los cuestionarios.

Finalmente, del análisis de las respuestas relacionadas con las medidas de fomento a la demanda de biomasa puede desprenderse la necesidad existente de potenciar la creación de un mercado estable de biomasa que asegure al usuario poder tener garantizado el suministro de combustible, así como su calidad y la estabilidad en el precio, como medida esencial para el despegue del sector. Con menor puntuación, se encuentran las medidas consistentes en el incremento de ayudas para la adquisición de instalaciones y la promoción pública para dar a conocer las ventajas de la bioenergía tanto en su uso como sobre el medio ambiente.

Tabla 8

Incentivos que podrían favorecer la demanda de bioenergía

\begin{tabular}{|l|c|c|}
\hline \multicolumn{1}{|c|}{ Incentivos } & $\begin{array}{c}\text { Valoración } \\
\text { media }\end{array}$ & $\begin{array}{c}\text { Desviación } \\
\text { típica }\end{array}$ \\
\hline $\begin{array}{l}\text { Establecer un mayor número de ayudas para la adquisición de } \\
\text { instalaciones }\end{array}$ & 2,53 & 1,08 \\
\hline $\begin{array}{l}\text { Potenciar la creación de un mercado estable de biomasa que } \\
\text { asegure al usuario poder tener garantizado el suministro de } \\
\text { combustible, así como su calidad y precio }\end{array}$ & 3,57 & 0,71 \\
\hline
\end{tabular}


Tabla 8 (continuación)

Incentivos que podrían favorecer la demanda de bioenergía

\begin{tabular}{|l|c|c|}
\hline \multicolumn{1}{|c|}{ Incentivos } & $\begin{array}{c}\text { Valoración } \\
\text { media }\end{array}$ & $\begin{array}{c}\text { Desviación } \\
\text { típica }\end{array}$ \\
\hline $\begin{array}{l}\text { Implantar, a modo de referencia, un mayor número de } \\
\text { instalaciones de biomasa en la Administración }\end{array}$ & 1,91 & 0,99 \\
\hline $\begin{array}{l}\text { Realizar una mayor difusión por parte de la Administración } \\
\text { acerca de las comodidades en el manejo de la biomasa así } \\
\text { como de sus ventajas ambientales }\end{array}$ & 2,19 & 1,10 \\
\hline
\end{tabular}

Fuente: Elaboración propia a partir de los resultados de los cuestionarios.

\section{ESTRATEGIAS PROPUESTAS PARA EL DESARROLLO DEL SECTOR DE LA BIOENERGÍA EN EL MEDIO RURAL}

A partir de las conclusiones extraídas de la consulta a los expertos del sector, así como de gran parte de la literatura al respecto, a continuación se proponen una serie de estrategias que podrían fomentar $\mathrm{y}$ favorecer al sector de la bioenergía y su difusión (producción y consumo) dentro de los territorios rurales, mejorando con ello el desarrollo socioeconómico de estas zonas. Gran parte de estas estrategias ya han sido propuestas en diferentes planes, normativas o mesas de trabajo, pero, por diversos factores (falta de financiación, falta de apoyo público...) no han logrado una mejora notoria en este sector. No obstante, la práctica totalidad de implicados coinciden en señalar la importancia que tiene el desarrollo de la bioenergía sobre la economía y el medio ambiente (como ya está ocurriendo en otros países) y que todos los indicios apuntan al desarrollo de este tipo de energía en los próximos años, adelantándose dicho proceso a medida que se tomen las medidas más adecuadas a la situación actual. En consecuencia, la estrategia a aplicar podría estar definida en torno a tres ejes: a) Diseño de un modelo agroindustrial de proximidad entre productores de materia prima y plantas transformadoras; b) Apoyo a la oferta a través de normativa y políticas favorables y c) Apoyo a la demanda a través de normativa y políticas favorables.

Diseño de un modelo agroindustrial de proximidad entre productores de materia prima y plantas transformadoras. La planificación de una logística adecuada es en muchas ocasiones la clave del éxito de buena parte de proyectos que se ponen en marcha. Haciendo referencia al sector que ocupa este estudio, se consideran esenciales iniciativas que promuevan la consecución del recurso a un precio adecuado para ambas partes y que aseguren también la materia prima de manera continuada, ya que ambos factores, como se ha comprobado en el anterior apartado, resultan vitales para el buen funcionamiento de este tipo de actividades. Para alcanzar este objetivo, se considera como medida eficaz la firma de acuerdos de suministro de materia prima con los productores, que garanticen tanto el compromiso de nivel de producción como de estabilidad de 
precios. En este sentido, una amplia parte de la literatura y las instancias públicas vienen apuntando en los últimos años que las iniciativas locales a pequeña escala, situadas cercanas a las zonas de producción primaria, facilitarían esta logística de suministro (Franco, 2007; Rodríguez y Sánchez, 2008; FAO, 2009), ya que la actividad transformadora estaría situada muy cercana a la fuente de materias primas, consiguiendo con ello otros beneficios adicionales sobre la zona (mejora de la competitividad agraria, aumento del empleo, aumento poblacional, etc.), tal y como se ha indicado con anterioridad. Para incentivar este modelo productivo, el movimiento asociativo y cooperativista desempeña un papel primordial, ya que este tipo de organizaciones facilita la defensa de intereses comunes y favorece la realización de contratos de suministro entre promotores y productores de biomasa, mediante la creación de una red de negocio y comercialización del producto.

Apoyo a la oferta a través de normativa y políticas favorables. Dado que el sector de la bioenergía en la actualidad es un sector emergente, se ha de potenciar su mercado a través de instrumentos que regulen y favorezcan tanto la oferta como la demanda del producto. En lo que concierne a la producción y tal y como se ha comprobado en el análisis de obstáculos anteriormente realizado, la desconfianza, el desconocimiento y los costes en concepto de inversión, investigación y adaptación de estructuras, conducen al fracaso de muchas iniciativas y al consiguiente estancamiento del sector. Así, por ejemplo, se podría mejorar la rentabilidad de las plantas transformadoras mediante instrumentos tales como: las subvenciones para la adquisición de equipos, las exenciones fiscales, los préstamos blandos o las ayudas destinadas a la investigación, entre otros, con el objeto de incentivar la oferta de bioenergía. En este sentido, se estima oportuna la potenciación de la investigación hacia métodos de recolección de ciertos tipos de biomasa que sean más eficientes y permitan una reducción de costes considerable. Adicionalmente, se considera interesante favorecer el procedimiento administrativo a seguir para la puesta en marcha de instalaciones transformadoras de biomasa, ya que las trabas y dificultades burocráticas suelen ser una queja general entre los promotores. Finalmente, sería necesario llevar a cabo acciones que favorezcan el intercambio de opiniones e impresiones entre todos los agentes implicados en la cadena de la bioenergía (agricultores, productores y consumidores) tales como visitas a plantas de producción bioenergética o mesas de trabajo que favorezcan el intercambio de ideas y de impresiones entre los distintos agentes, intentando con ello satisfacer sus necesidades. Existe otro tipo de agentes que pueden representar un papel fundamental como intermediadores entre la oferta y la demanda, como por ejemplo los fabricantes de automóviles, que podrían respaldar el consumo de biocarburantes y ayudar así a superar los recelos de los consumidores. 
Apoyo a la demanda a través de normativa y políticas favorables. Desde este punto de vista, se precisa atraer al usuario hacia la utilización de bioenergía, para lo cual resultarían sumamente útiles las campañas de promoción del recurso, tanto a la población en general como en los centros educativos, con el objetivo de dar a conocer las posibilidades que ofrece este sector desde el punto de vista de sus distintos usos, así como los impactos positivos de su implantación sobre los territorios rurales en especial. Asimismo, se necesita una mayor información dirigida a los potenciales usuarios acerca de la localización de los distribuidores de distintos productos bioenergéticos (biocombustibles, calderas de biomasa y pelets, etc...), ya que en ocasiones este desconocimiento es un factor que reduce el consumo del producto. Por su parte, podrían resultar medidas positivas las reducciones fiscales sobre el consumo de este tipo de energía y las ayudas para la adquisición o renovación de equipos domésticos que necesiten biomasa para su funcionamiento.

\section{CONCLUSIONES}

La generación de energía a través del aprovechamiento de biomasa puede suponer una estrategia adecuada para la dinamización socioeconómica de muchas zonas rurales que en los últimos años vienen reflejando una importante descapitalización productiva y humana. La bioenergía se configura, consecuentemente, como un sector capaz de generar importantes impactos sobre el desarrollo sostenible de las áreas rurales, atendiendo tanto a la dimensión económica, como a la social y a la ambiental.

Sin embargo, debido a factores variados, la expansión de este sector y su influencia positiva sobre las zonas rurales no se está produciendo como era de esperar. Lejos de la creencia generalizada de no hace muchos años de que los cultivos energéticos podrían suponer una firme solución a la totalidad de los problemas de la agricultura menos competitiva de las zonas rurales del interior peninsular, actualmente tampoco están proliferando otras iniciativas de carácter más local. Esta infrautilización de los recursos biomásicos está provocando que no se cumplan los objetivos cuantitativos en cuanto a consumo de renovables adquiridos por el gobierno español a escala europea.

Los expertos consultados en el presente estudio destacan que la falta de éxito de este tipo de iniciativas está relacionada con la inexistencia de un mercado organizado de biomasa, junto con el desconocimiento por parte de agricultores y promotores empresariales acerca de las posibilidades de este recurso para construir diversos tipos de instalaciones de producción bioenergética. A estos obstáculos hay que añadir las reticencias de los potenciales usuarios a la hora de consumir bioenergía para sus distintos usos, dada la falta de apoyo y promoción institucional que fomente el uso de este tipo de energía, lo que repercute sobre 
la falta de información de gran parte de la población acerca de las posibilidades de esta fuente energética.

A la luz de estos datos, parece desprenderse que no hay una relación demasiado congruente entre las bonanzas del sector de la bioenergía en pro del desarrollo rural que otorgan tanto la mayor parte de las investigaciones al respecto como las diversas instituciones y las acciones que finalmente se están llevando a cabo para favorecer de forma definitiva tanto su producción como su consumo. Sin embargo, los beneficios que podrían generar este tipo de iniciativas sobre las zonas rurales son muy significativos, tal y como lo han puesto de manifiesto el grupo de expertos entrevistado.

Para alcanzar esos efectos positivos, se hace necesario un verdadero compromiso público hacia el apoyo a este sector. Como consecuencia, se propone el diseño de un modelo agroindustrial en el que exista una estrecha relación de proximidad o incluso de propiedad entre los productores de la materia prima y los transformadores, y todo ello complementado con la actuación del sector asociativo y cooperativo, como estrategia adecuada para que los efectos económicos positivos que podría generar este sector, recaigan sobre el desarrollo del medio rural en el que se encuentre localizada la industria. Se considera también fundamental la vinculación de la propia población rural en este tipo de proyectos, a través de su integración ya sea como productores (agricultores, socios de plantas transformadoras...) o como consumidores del producto, incentivando con ello el autoabastecimiento energético a escala local o comarcal.

\section{REFERENCIAS BIBLIOGRÁFICAS}

ADAS CONSULTING (2003). Renewable energy and its impact on rural development and sustainability in the UK. Wolverhampton: ADAS Consulting Ltd. and University of Newcastle.

ASOCIACIÓN DE PRODUCTORES DE ENERGÍAS RENOVABLES (APPA) (2010). Estudio del impacto macroeconómico de las energías renovables en España año 2009. Asociación de Productores de Energías Renovables. http://www.appa.es. [Último acceso: Enero de 2011].

ASOCIACIÓN NACIONAL DE EMPRESAS FORESTALES (ASEMFO) (2007). Guía para el uso y aprovechamiento de la biomasa en el sector forestal. http://www.asemfo.org. [Último acceso: Enero de 2011]

BROUWER, F. (ed.) (2004). Sustaining agriculture and the rural environment, governance, policy and multifunctionality. Massachussets: Edward Elgar publishing. 
BURGUILLO, M. Y DEL RÍO, P. (2008). "La contribución de las energías renovables al desarrollo rural sostenible en la Unión Europea: Pautas teóricas para el análisis empírico". Información Comercial Española, Revista de Economía, 845, 149-165.

CARPINTERO, O. (2006). "Biocombustibles y uso energético de la biomasa: un análisis crítico". El ecologista, 49, 20-26.

COMISIÓN EUROPEA (1988). El futuro del mundo rural. Comunicación de la Comisión al Consejo y al Parlamento COM (88) 501 final.

COMISIÓN EUROPEA (1996). Declaración de Cork: un campo vivo. http://www.europa.eu. [Último acceso: Junio de 2010].

COMISIÓN EUROPEA (2003). Planting seeds for rural futures. Segunda Conferencia Europea sobre Desarrollo Rural. Salzburgo (Austria). http://www.europa.eu. [Último acceso: Junio de 2010].

COMISIÓN EUROPEA (2009). Informe de avance sobre la energía procedente de fuentes renovables. COM(2009) 192 final. Comisión de las Comunidades Europeas.

CONSEJO ECONÓMICO Y SOCIAL (CES) de Castilla y León (2009). Expectativas del sector de la bioenergía en Castilla y León. Valladolid: CES de Castilla y León.

ECOLOGISTAS MARTXAN (2002). Incidencia ambiental del empleo de biomasa con fines energéticos. Iruña: Ecologistas Martxan.

DEL RÍO, P. Y BURGUILLO, M. (2008). "Assessing the impact of renewable energy deployment on local sustainability: towards a theoretical framework". Renewable and sustainable energy reviews, 12(2008), 1.325-1.344.

DINCER, I. (2000). "Renewable energy and sustainable development: a crucial review". Renewable and Sustainable Energy Reviews, 4 (2), 157-175.

FAO (2008). Bioenergía, seguridad y sostenibilidad alimentaria: los desafíos del cambio climático y la bioenergía. Roma: Food and Agriculture Organisation of the United Nations and Policy Innovation Systems for Clean Energy Security.

FAO (2009). Small-Scale Bioenergy Initiatives: brief description and preliminary lessons on livelihood impacts from case studies in Asia, Latin America and Africa. Rome: Food and Agriculture Organisation of the United Nations and Policy Innovation Systems for Clean Energy Security.

FAULIN, J.; LERA, F.; PINTOR, J. Y GARCÍA, J. (2006). "The outlook for renewable energy in Navarre: an economic profile". Energy Policy, 34 (15), 2.201-2.216.

FRANCO, F. (2007). Agricultura y despoblación rural en Palencia. Palencia: Publicaciones Institución Tello Téllez de Meneses, 78.

FUNDACIÓN ENCUENTRO (2007). "Realidad productiva rural y escenarios de futuro". En Fundación Encuentro: Informe España 2007 (pp. 113-191). Madrid: Fundación Encuentro. 
GARCÍA PASCUAL, F. (coord.) (2003). La lucha contra la despoblación todavía necesaria: políticas y estrategias sobre la despoblación en las áreas rurales del siglo XXI. Jaca (Huesca): Centro de Estudios sobre la Despoblación y el Desarrollo de Áreas Rurales.

GARCÍA SANZ, B. E IZCARA, S. (2000). "Pluriactividad y diversificación de ingresos en el medio rural". Sociología del trabajo, nueva época, 38, 119134.

GÓMEZ-LIMÓN, J.A.; ATANCE, I. Y RICO, M. (2007). "Percepción pública del problema de la despoblación del medio rural en Castilla y León". AGER. Revista de Estudios sobre Despoblación y Desarrollo, 6, 9-60.

GONZÁLEZ REGIDOR, J. (2008). Desarrollo rural sostenible: un nuevo desafío. Madrid: Ministerio de Medio Ambiente y Medio rural y Marino.

JUNTA DE CASTILLA Y LEÓN (2010). Plan Regional de Ámbito Sectorial de la Bioenergía de Castilla y León. Ente Regional de la Energía en Castilla y León. http://www.jcyl.es. [Último acceso: Enero de 2011].

LÓPEZ, L.M.; SALA, J.; GRANADA, E. Y JUÁREZ, M. (2006). "Contribution of renewable energy sources to electricity production in the La Rioja Autonomous Community, Spain. A review". Renewable and Sustainable Energy Reviews, 11 (6), 1.244-1.259.

MEIER, P. Y MUNASINGHE, M. (2004). Sustainable energy in developing countries. Policy analysis and case studies. Cheltenham: Edward Elgar.

MíGUEZ, J.L.; LÓPEZ, L.; SALA, J.; PORTEIRO, J.; GRANADA, E.; MORÁN, J. Y JUÁREZ, M. (2006). "Review of compliance with EU-2010 targets on renewable energy in Galicia (Spain)". Renewable and Sustainable Energy Reviews, 10 (3), 225-247.

MOYANO, E. Y GARRIDO, F.E. (2007). "A propósito de la multifuncionalidad. Discursos y políticas sobre agricultura y desarrollo rural". En Gómez-Limón, J.A. y Barreiro, J. (coords.): La multifuncionalidad de la agricultura en España (pp. 59-75). Madrid: Ministerio de Agricultura, Pesca y Alimentación.

ORTUÑO, S.F.; FERNÁNDEZ-CAVADA, J.L. Y CASADO, J. (2010). "La bioenergía y el desarrollo rural". VIII Coloquio Ibérico de Estudios Rurales. Cáceres.

RICO, M. Y GÓMEZ-LIMÓN, J.A. (2008). "Sociedad y desarrollo rural en Castilla y León: un estudio de opinión pública". Boletín de la Asociación de Geógrafos Españoles, 48, 199-223.

RODRÍGUEZ, F. Y SÁNCHEZ, J.I. (2008). "Los cultivos energéticos en Castilla y León”. En Gómez-Limón, J.A. (coord.): El futuro de la agricultura en Castilla y León (pp. 79-95). Palencia: Caja España e Itagra ct.

VAN HUYLENBROECK, G. Y DURAND, G. (coords.) (2003). Multifunctionality: A new paradigm for European agricultura. Aldershot: Ashgate. 
